\title{
Outlier Preservation by Dimensionality Reduction Techniques
}

\author{
Martijn Onderwater ${ }^{1,2, *}$ \\ ${ }^{1}$ Center for Mathematics and Computer Science (CWI) \\ Science Park 123, 1098 XG, Amsterdam, \\ The Netherlands. \\ E-mail: m.onderwater@cwi.nl \\ Fax: +31 (0)20 5924199 \\ ${ }^{2}$ VU University, Faculty of Sciences \\ Amsterdam, The Netherlands. \\ ${ }^{*}$ Corresponding author
}

September 24, 2013

IJDATS - 8740

\begin{abstract}
Sensors are increasingly part of our daily lives: motion detection, lighting control, and energy consumption all rely on sensors. Combining this information into, for instance, simple and comprehensive graphs can be quite challenging. Dimensionality reduction is often used to address this problem, by decreasing the number of variables in the data and looking for shorter representations. However, dimensionality reduction is often aimed at normal daily data, and applying it to events deviating from this daily data (so-called outliers) can affect such events negatively. In particular, outliers might go unnoticed.In this paper we show that dimensionality reduction can indeed have a large impact on outliers. To that end we apply three dimensionality reduction techniques to three real-world data sets, and inspect how well they preserve outliers. We use several performance measures to show how well these techniques are capable of preserving outliers, and we discuss the results.
\end{abstract}


Keywords: dimensionality reduction; outlier detection; multidimensional scaling; principal component analysis; t-stochastic neighbourhood embedding; peeling; F1-score; Matthews Correlation; Relative Information Score; sensor network.

Biographical notes: Martijn Onderwater is a Ph.D. student at the Center for Mathematics and Computer Science (Amsterdam, The Netherlands), and at VU University (Amsterdam, The Netherlands). He received a M.Sc. degree in Mathematics (2003) from the University of Leiden (The Netherlands), and a M.Sc. degree in Business Mathematics \& Informatics (2010, Cum Laude) from VU University Amsterdam. He also has several years of commercial experience as a software engineer. His research focuses on sensor networks, and his interests include dimensionality reduction, outlier detection, caching in sensor networks, Markov decision processes, correlations in sensor data, and middleware for sensor networks.

\section{Introduction}

Recent technological developments have resulted in a broad range of cheap and powerful sensors, enabling companies to use sensor networks in a cost-effective way. Consequently, sensor networks will increasingly become part of our daily life. One can for instance envision a house with sensors related to smoke detection, lighting control, motion detection, environmental information, security issues, and structural monitoring.

Combining all this information to actionable insights is a challenging problem. For instance, in the event of a burglary in a house, the sensors involved in motion detection, environmental monitoring, and security all yield useful information. Providing a short insightful summary that helps users identify the event and take appropriate action is essential. Dimensionality reduction (DR) is a family of techniques aimed at reducing the number of variables (dimensions) in the data and thus making the data set smaller. In essence, it helps identify what is important, and what is not.

However, in practise dimensionality reduction often yields some loss of information, and applications might be affected by this loss. For instance, the burglary mentioned before is a (hopefully) rare event that is different from normal patterns in the sensor data (i.e. a so-called outlier). Unfortunately, DR-methods often lose outliers among the regular sensor data. Figure 1 illustrates this situation using a two-dimensional data set with an outlier near the top-left corner. When dimensionality is reduced by projecting all points onto a line, the outlier is mapped into the center of the reduced data set (the middle arrow in Figure 1 ), and is thus no longer an outlier. So dimensionality reduction might lose outliers among regular points, causing problems for applications relying on the 


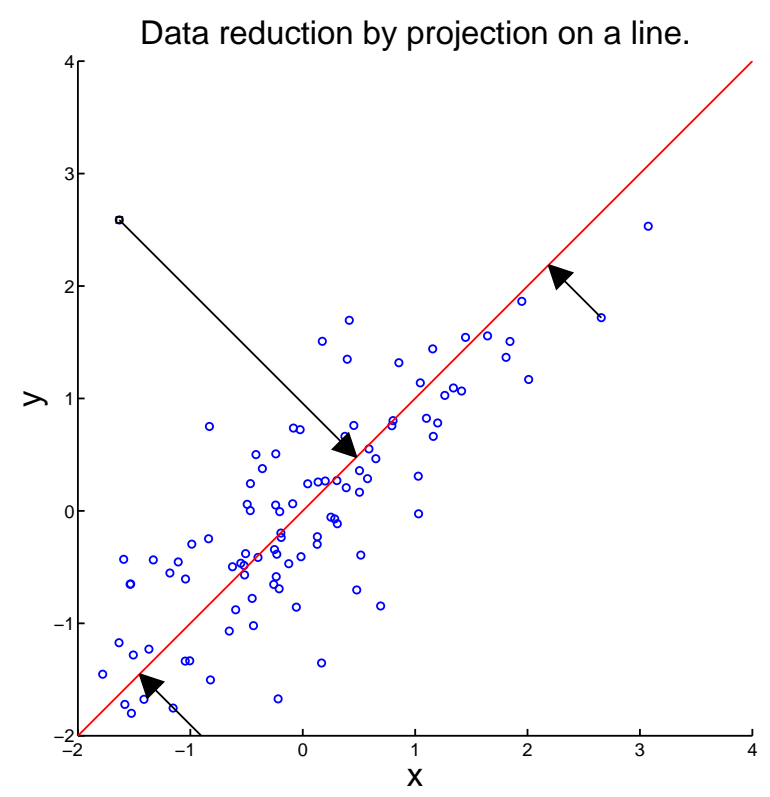

Figure 1. A two-dimensional data set reduced to one dimension, with an outlier (middle arrow) mapped to the center of the reduced data set

detection of outliers. The example in Figure 1 illustrates one DR-technique, but many others exists, each affecting outliers differently.

In this paper we show that DR-techniques affect outliers, by measuring their capability to preserve outliers. For this purpose we describe three well-known DR-techniques that are relevant for a broad audience, and apply them to several real-world data sets from a sensor-related context. For each DR-technique we capture its capability to preserve outliers in three performance measures, and compare the results. From the three techniques we will identify the one with the best performance, and discuss the intuitions behind the scores.

Research on both dimensionality reduction and outlier detection is abundant. An overview of dimensionality reduction techniques can be obtained from CarreiraPerpinán (1997); Fodor (2002); Gupta and Kapoor (2012); Kline and Galbraith (2009); Onderwater (2010); Van der Maaten et al. (2009). For outlier detection, we refer readers to, e.g., Agyemang et al. (2006); Hodge and Austin (2004); Maalouf and Trafalis (2011); Zhang et al. (2010, 2007). Certain specific topics such as intrusion detection Gogoi et al. (2011) and fraud detection Becker et al. (2010); Phua et al. (2005) are closely related to outlier detection. In Muñoz and Muruzábal (1998) the authors consider Kohonen's Self Organizing Maps (som, Kohonen (2001)) and how this DR-technique can be used to identify outliers. Harmeling et al. (2006) illustrate the effect of outlier-removal on Isomap (Tenen- 
baum et al. (2000)), another DR-technique. Chakrabarti and Mehrotra (2000) look at local DR, where reduction is applied to previously identified clusters. Outlier detection occurs as part of the cluster-identification phase. Non of these papers, however, look at outlier preservation by DR-techniques, as discussed in this paper. In Escalante (2005), the authors compare multiple outlier detection methods on various data sets, including one data set with its dimensionality reduced. As in our paper, their analysis also suggests that outlier detection is affected by dimensionality reduction, although they only use one DR-methods and one performance measure. The paper by Nguyen et al. (2010) has a setup that is close to our approach: four DR-methods (feature extraction methods in their terminology) are applied to three data sets, and the performance (using one score measure) is inspected for two outlier detection methods. However, their DR-methods are selected from the Feature Extraction domain, and are not well-known in the DR-community.

The structure of the paper is as follows: Section 2 describes the DR-techniques, Section 3 contains the outlier detection method as well as the performance measures. Then, in Section 4 we describe the data sets that we use in the experiments. Section 5 shows the output of the experiments and discusses the results, followed by conclusions, recommendations, and ideas for further research in Section 6.

\section{Dimensionality reduction techniques}

Denote by $n$ the number of measurements and by $d$ the number of sensors producing the measurements. The number of sensors is known as the dimension of the data, and DR-techniques aim to lower this dimension to a smaller value. More formally, if the measurements are vectors $\mathbf{x}_{1}, \ldots, \mathbf{x}_{n} \in \mathbb{R}^{d}$, then DR-techniques try to find points $\mathbf{y}_{1}, \ldots, \mathbf{y}_{n} \in \mathbb{R}^{d^{\prime}}$ with $d^{\prime}<d$. Dimensionality reduction is often used for, e.g., visualisation $\operatorname{Li}(1992)$;Tsai (2012), as a preprocessing step for further analysis UmaMaheswari and Rajaram (2009); Garg and Murty (2009); Ravi and Pramodh (2010), or for computational efficiency Hahn et al. (2003); Dai et al. (2006).

This section describes three well-known and often used DR-techniques: Principal Component Analysis (PCA), Multidimensional Scaling (MDS), and t-Stochastic Neighbourhood Embedding (t-SNE). 


\subsection{Principal Component Analysis}

Principal Component Analysis was initially proposed by Pearson (1901). It finds a low dimensional representation of the data with minimal loss of variation in the reduced data set. The first step in PCA is a linear change of base from $\mathbf{x}_{1}, \ldots, \mathbf{x}_{d} \in \mathbb{R}^{d}$ to $\mathbf{u}_{1}, \ldots, \mathbf{u}_{d} \in \mathbb{R}^{d}$, where $\mathbf{u}_{1}$ is aligned with the direction of maximum variance in the data. Vectors $\mathbf{u}_{i}(2 \leq i \leq d)$ are also lined up with the direction of maximum variance, but constrained to be perpendicular to $\mathbf{u}_{i-1}, \ldots, \mathbf{u}_{1}$. The $\mathbf{u}_{i}$ vectors are called Principal Components.

Suppose that the $n$ data points are in the $n \times d$ matrix $X$, then the vectors $\mathbf{u}_{i}$ are found by calculating the eigenvectors of the correlation matrix $(C)$ (Johnson and Wichern (2002)). The eigenvalues of $C$ correspond to the amount of variance explained by the corresponding eigenvectors. Dimensionality reduction is achieved by omitting eigenvectors $\mathbf{u}_{d^{\prime}+1}, \ldots \mathbf{u}_{d}$ once eigenvalues $\lambda_{1}, \ldots, \lambda_{d^{\prime}}$ explain enough of the variance of the data set. Summarized, the process works as follows:

- Construct the data matrix $X$.

- Compute the correlation matrix $C$.

- Find the eigenvalues and eigenvectors of $C$.

- Determine $d^{\prime}$ such that $\lambda_{1}, \ldots, \lambda_{d^{\prime}}$ explain enough of the variance of the data.

- Construct matrix $\hat{U}=\left[\mathbf{u}_{1} \ldots \mathbf{u}_{d^{\prime}}\right]$.

- Reduce dimension by computing $\hat{X}=X \hat{U}^{T}$.

More details on PCA can be found in, e.g., Härdle and Simar (2012); Johnson and Wichern (2002); Lattin et al. (2003); Tabachnick and Fidell (2001); Yoon (2003).

\subsection{Multidimensional Scaling}

Multidimensional Scaling is the name for a family of dimensionality reduction techniques based on preserving distances in the data set. The classical version of Multidimensional Scaling finds points $\mathbf{y}_{1}, \ldots, \mathbf{y}_{n} \in \mathbb{R}^{d^{\prime}}$ in a low dimensional space that minimize

$$
\min _{\mathbf{y}_{1}, \ldots, \mathbf{y}_{n}} \sum_{i=1}^{n} \sum_{j=1}^{n}\left(\left\|\mathbf{x}_{i}-\mathbf{x}_{j}\right\|-\left\|\mathbf{y}_{i}-\mathbf{y}_{j}\right\|\right)^{2}
$$


Here $\mathbf{x}_{1}, \ldots, \mathbf{x}_{n} \in \mathbb{R}^{d}$ are the high dimensional points, and $\|\cdot\|$ is the Euclidean distance in the respective space. The classical version of MDS is equivalent to PCA, see for instance Ghodsi (2006). Other members of the MDS family use a different distance measure or a different quantity to optimize than Eq. (1). We use a version of MDS with the so-called squared stress criterion

$$
\min _{\mathbf{y}_{1}, \ldots, \mathbf{y}_{n}} \frac{\sum_{i=1}^{n} \sum_{j=1}^{n}\left(\left\|\mathbf{x}_{i}-\mathbf{x}_{j}\right\|^{2}-\left\|\mathbf{y}_{i}-\mathbf{y}_{j}\right\|^{2}\right)^{2}}{\sum_{i=1}^{n} \sum_{j=1}^{n}\left\|\mathbf{x}_{i}-\mathbf{x}_{j}\right\|^{4}} .
$$

For the distance measure $\left\|\mathbf{x}_{i}-\mathbf{x}_{j}\right\|$ we do not use the Euclidean distance measure as in the classical version of MDS. To see why, note that MDS with the Euclidean distance is sensitive to natural variations in the data. Consider, for instance, a data set consisting of two columns, one with values uniformly drawn from $[1000-2000)$ and one with values drawn from $[0,1)$. Clearly, all values in the first column are several orders of magnitude larger than those in the second column. When minimizing the quantity in Eq. (1) the procedure focuses on the elements of the first column, since that brings it closest the minimum. In essence, the second column is ignored and MDS is biased towards the first column.

To overcome this problem the Euclidean distance is replaced by the Mahalanobis distance (Mahalanobis (1936))

$$
\left\|x_{i}-x_{j}\right\|_{M}=\sqrt{\left(\mathbf{x}_{\mathbf{i}}-\mathbf{x}_{\mathbf{j}}\right) \Sigma^{-1}\left(\mathbf{x}_{\mathbf{i}}-\mathbf{x}_{\mathbf{j}}\right)^{T}},
$$

where $\Sigma$ is the covariance matrix. By including the covariance matrix in the distance measure, the natural variations in the data are removed and thus MDS is unbiased with respect to dimensions. Eq. (1) then becomes

$$
\min _{\mathbf{y}_{1}, \ldots, \mathbf{y}_{n}} \frac{\sum_{i=1}^{n} \sum_{j=1}^{n}\left(\left\|\mathbf{x}_{i}-\mathbf{x}_{j}\right\|_{M}^{2}-\left\|\mathbf{y}_{i}-\mathbf{y}_{j}\right\|^{2}\right)^{2}}{\sum_{i=1}^{n} \sum_{j=1}^{n}\left\|\mathbf{x}_{i}-\mathbf{x}_{j}\right\|_{M}^{4}} .
$$

Note that the Mahalanobis distance is only used for the high-dimension points $\mathbf{x}_{i}$, because the low-dimensional points $\mathbf{y}_{i}$ are found by the minimization. 


\section{$2.3 \quad$ t-Stochastic Neighbourhood Embedding}

\subsubsection{Stochastic Neighbourhood Embedding}

t-Stochastic Neighbourhood Embedding is a variation on Stochastic Neighbourhood Embedding (SNE), first proposed by Hinton and Roweis (2002). SNE presents the novel idea of defining a probability that two points are neighbours. Mapping to low dimensional space is achieved by choosing points that preserve these probabilities. They define Gaussian-inspired probabilities $p_{i \mid j}$ in high dimensional space, representing the probability of point $\mathbf{x}_{i}$ being a neighbour of a given point $\mathbf{x}_{j}$, as

$$
p_{i \mid j}=\frac{e^{-\left\|\mathbf{x}_{i}-\mathbf{x}_{j}\right\|_{M}^{2} / 2 \sigma_{i}^{2}}}{\sum_{k \neq i} e^{-\left\|\mathbf{x}_{i}-\mathbf{x}_{k}\right\|_{M}^{2} / 2 \sigma_{i}^{2}}} .
$$

The parameter $\sigma_{i}$ is set by hand or determined with a special search algorithm. Note how we again employ the Mahalanobis distance for the high-dimensional points. In low dimensional space, probabilities similar to those in Eq. (5) are defined as

$$
q_{i \mid j}=\frac{e^{-\left\|\mathbf{y}_{i}-\mathbf{y}_{j}\right\|^{2}}}{\sum_{k \neq i} e^{-\left\|\mathbf{y}_{i}-\mathbf{y}_{k}\right\|^{2}}}
$$

The parameter $\sigma_{i}$ is not necessary here, because it would only lead to a rescaling of the resulting low dimensional points $\mathbf{y}_{i}$. The $\mathbf{y}_{i}$ are then found by minimizing the Kullback-Leibler divergence of these two probability distributions

$$
\min _{\mathbf{y}_{1}, \ldots, \mathbf{y}_{n}} \sum_{i} \sum_{j} p_{i \mid j} \log \frac{p_{i \mid j}}{q_{i \mid j}}
$$

Minimization of Eq. (7) can be done with, e.g., the gradient descent algorithm, or the scaled conjugate gradients procedure.

\subsection{2 t-SNE}

In Van der Maaten and Hinton (2008) the authors propose t-SNE, which differs from SNE in two aspects. First, note that the probabilities in Eq. (5) are not necessarily symmetric, i.e., $p_{i \mid j}$ and $p_{j \mid i}$ do not need to be equal. This 
complicates minimization of Eq. (7), because it has twice as many variables as in the symmetric case. In t-SNE, these probabilities are redefined to be symmetric:

$$
p_{i j}=\frac{p_{i \mid j}+p_{j \mid i}}{2 n}
$$

Additionally, this ensures that $\sum j p_{i j}>1 / 2 n$ so that each point (including outliers) have a significant contribution to the cost function. The second change proposed for t-SNE concerns the $q_{i j}$. Instead of using Gaussian-style probabilities as in Eq. (6), t-SNE uses probabilities inspired by the Student t-distribution (with one degree of freedom):

$$
q_{i j}=\frac{\left(1+\left\|\mathbf{y}_{i}-\mathbf{y}_{j}\right\|^{2}\right)^{-1}}{\sum_{k \neq i}\left(1+\left\|\mathbf{y}_{i}-\mathbf{y}_{k}\right\|^{2}\right)^{-1}} .
$$

This distribution has heavier tails than the Gaussian used by SNE, so should map nearby high dimensional points less nearby in low dimensional space than SNE. A justification for this approach comes from the so-called Crowding problem: there is much more room in high dimensional space for points, so in a low dimensional representation data points tend to be 'squeezed' together. By using the Student t-distribution, these crowded points are placed just a bit further apart. Low dimensional points are still found by optimizing the Kullback-Leibler divergence

$$
\min _{\mathbf{y}_{1}, \ldots, \mathbf{y}_{n}} \sum_{i} \sum_{j} p_{i j} \log \frac{p_{i j}}{q_{i j}} .
$$

\section{$3 \quad$ Experimental setup}

We adopt the following experimental setup when investigating dimensionality reduction for outlier preservation:

1. Modify each data set so that it has zero mean and unit variance. This is a common preprocessing step for experimental data.

2. Find outliers in the high-dimensional (centered and scaled) data set.

3. Reduce the data set to two dimensions.

4. Again look for outliers, this time in the low-dimensional data. 
5. Compute a score showing how each DR-methods performs on the data set.

We apply this setup to the DR-techniques from Section 2 and to a number of realworld data sets, described later in Sections 4.1-4.3. The sections below describe the technique that we use for outlier detection, and three performance measures that we use to assess how well outliers are preserved. For the DR-techniques we used Matlab implementations available in the Dimensionality Reduction Toolbox by Van der Maaten (2009).

\subsection{Onion Peeling}

The idea of Onion Peeling, or Peeling in short, is to construct a convex hull around all the points in the data set and then find the points that are on the convex hull. These points form the first 'peel' and are removed from the data set. Repeating the process gives more peels, each containing a number of points.

This technique can be modified for finding outliers. The largest outlier in the data set is on the first peel, so by inspecting the total distance of each point on the hull to all other points in the data set, we can find the one with the largest total distance. Removing this point from the data set and repeating the process gives new outliers. The decrease in volume of the convex hull after removing an outlier is used as a stop criterion. Once the volume decreases by a fraction less than $\alpha(0 \leq \alpha \leq 1)$, we stop looking for outliers. Although with this criterion there is no guarantee that all outliers are found, it does assure that all found points are outliers. In our experiments we set $\alpha=0.005$. Peeling is outlined in algorithm 1.

Algorithm 1: Peeling

1. Calculate the convex hull around all the points in the data set.

2. Find the point on the hull with the largest (Mahalanobis) distance to all other points in the data set.

3. Remember the outlier and remove it from the data set.

4. Calculate the new convex hull, and check if the stop criterion is reached. If so, stop, otherwise continue with step 2.

\section{$3.2 \quad$ Measuring performance}

After running the experiment for one data set and one DR-method, we need to quantify the performance of this method with respect to the preservation of outliers. In order to do so, we assign each point to one of four groups: 
- True Positive (TP). The point is an outlier both before and after DR.

- False Positive (FP). The point is not an outlier before DR, but is one after.

- False Negative (FN). The point is an outlier before DR, but not after.

- True Negative (TN). The point is not an outlier before DR, nor after.

We can summarize these quantities in a confusion matrix, as shown in Figure 2. In an ideal scenario the confusion matrix would be diagonal (i.e. 0 FPs and FNs), indicating that all outliers and non-outliers were correctly retained by the DR-methods. However, in practise the matrix will often contain some FPs and FNs, and the performance of a DR-methods is judged by all four quantities.

Outlier

before DR?

\begin{tabular}{|c|c|c|c|}
\cline { 3 - 4 } \multicolumn{1}{c|}{} & & Yes & No \\
\cline { 2 - 4 } After DR? & Yes & TP & FP \\
\cline { 2 - 4 } & No & FN & TN \\
\cline { 2 - 4 } & & &
\end{tabular}

Figure 2. Confusion matrix showing what happened to outliers after DR

Confusion matrices are used in several research communities to asses the performance of, e.g., binary classifiers and statistical tests. Often a single number is needed to capture performance, which subsequently results in a combination of the four quantities in the table. Several such combinations exist and are used in various fields of research, see the overview in paper Powers (2011).

We intend to describe three performance measures that are often used in the literature, but before we do so we highlight one complicating aspect of our problem scenario. Since we deal with outliers, most practical data sets will have a significantly larger number of non-outliers than outliers. Hence, in the confusion matrix the TN will usually be the largest number. As an example of a performance measure that is affected by this, we look at accuracy, defined as

$$
\text { accuracy }=\frac{\mathrm{TP}+\mathrm{TN}}{\mathrm{TP}+\mathrm{FP}+\mathrm{FN}+\mathrm{TN}} .
$$

Since $\mathrm{TN}$ is dominating number, accuracy will always be close to 1, making it difficult to identify small differences in performance. Hence, the three performance measures that are described below are selected because they are capable of handling this issue. 


\subsubsection{F1-score}

The F1-score is a combination of recall and performance:

- Recall. The fraction of high-dimensional outliers retained by the DRmethods (i.e. $\mathrm{TP} /(\mathrm{TP}+\mathrm{FN}))$, which is maximized for $\mathrm{FN}=0$.

- Precision. The fraction of low-dimensional outliers that were also highdimensional outliers (i.e. $\mathrm{TP} /(\mathrm{TP}+\mathrm{FP})$ ), which is maximized for $\mathrm{FP}=0$.

The F1-score takes the harmonic mean of precision and recall, resulting in a number between 0 (when $\mathrm{TP}=0)$ and $1($ when $\mathrm{FP}=\mathrm{FN}=0)$ :

$$
\begin{aligned}
F 1 & =2 \cdot \frac{\text { precision } \cdot \text { recall }}{\text { precision }+ \text { recall }} \\
& =2 \cdot \frac{\mathrm{TP} /(\mathrm{TP}+\mathrm{FP}) \cdot \mathrm{TP} /(\mathrm{TP}+\mathrm{FN})}{\mathrm{TP} /(\mathrm{TP}+\mathrm{FP})+\mathrm{TP} /(\mathrm{TP}+\mathrm{FN})} \\
& =\frac{2 \mathrm{TP}}{(2 \mathrm{TP}+\mathrm{FN}+\mathrm{FP})} .
\end{aligned}
$$

If $\mathrm{TP}+\mathrm{FN}=0$ or $\mathrm{TP}+\mathrm{FP}=0$ then the F1-score is defined as 0 . Note that the element TN of the confusion table does not affect the score, and it is therefor not affected by the sparsity of outliers. The F1-score is used in, e.g., Information Retrieval Cao et al. (2009); Martins et al. (2010) and Machine Learning Escalante (2005); Sha and Pereira (2003); Valstar et al. (2011).

\subsubsection{Matthews Correlation}

The Matthews Correlation (due to Matthews (1975)) computes a correlation coefficient between the class labels (i.e. outlier or non-outlier) in high and low dimension of each point in the data sets. It results in a number between -1 (perfect anti-correlation) and 1 (perfect correlation), with 0 indicating the absence of correlation. Below we will derive an expression for the Matthews Correlation in terms of the elements of the confusion matrix. Define

$$
h_{i}= \begin{cases}1 & \text { if point } i \text { is an outlier in high dimension } \\ 0 & \text { otherwise }\end{cases}
$$

and similarly 


$$
l_{i}= \begin{cases}1 & \text { if point } i \text { is an outlier in low dimension } \\ 0 & \text { otherwise }\end{cases}
$$

With the $l_{i}$ and $h_{i}$ we can compute the correlation between the class labels $l_{1} \cdots l_{N}$ and $h_{1} \cdots h_{N}$ ( $N$ is the total number of points in the data set). The correlation can be interpreted as a measure of how well outliers are preserved.

This correlation $\rho$ is computed from

$$
\rho=\frac{1}{N-1} \frac{\sum_{i=1}^{N}\left(l_{i}-\bar{l}\right)\left(h_{i}-\bar{h}\right)}{\sigma_{l} \sigma_{h}}
$$

where

$$
\bar{l}=\frac{1}{N} \sum_{i} l_{i}=\frac{\mathrm{TP}+\mathrm{FP}}{N}, \quad \bar{h}=\frac{1}{N} \sum_{i} h_{i}=\frac{\mathrm{TP}+\mathrm{FN}}{N}
$$

using notation from the confusion matrix. The $\sigma_{l}$ is the standard deviation of the $l_{i}$, i.e.,

$$
\begin{aligned}
\sigma_{l} & =\sqrt{\frac{1}{N-1} \sum_{i}\left(l_{i}-\bar{l}\right)^{2}} \\
& =\sqrt{\frac{1}{N-1}} \sqrt{\sum_{i}\left(l_{i}^{2}-2 l_{i} \bar{l}+\bar{l}^{2}\right)} \\
& =\sqrt{\frac{1}{N-1}} \sqrt{\sum_{i}\left(l_{i}-2 l_{i} \bar{l}+\bar{l}^{2}\right)} \\
& =\sqrt{\frac{1}{N-1}} \sqrt{N \bar{l}-2 N \bar{l}^{2}+N \bar{l}^{2}} \\
& =\sqrt{\frac{N}{N-1}} \sqrt{\bar{l}(1-\bar{l})} .
\end{aligned}
$$

Similarly, the standard deviation of the $h_{i}$ becomes $\sigma_{h}=\sqrt{\frac{N}{N-1}} \sqrt{h(1-\bar{h})}$. Substituting these quantities in the correlation yields 


$$
\begin{aligned}
\rho & =\frac{\sum_{i=1}^{N}\left(l_{i}-\bar{l}\right)\left(h_{i}-\bar{h}\right)}{\sigma_{l} \sigma_{h}} \\
& =\frac{\sum_{i=1}^{N}\left(l_{i}-\bar{l}\right)\left(h_{i}-\bar{h}\right)}{N \sqrt{\overline{l h}(1-\bar{l})(1-\bar{h})}} \\
& =\frac{\sum_{i=1}^{N}\left(l_{i} h_{i}-\bar{l} h_{i}-l_{i} \bar{h}+\overline{l h}\right)}{N \sqrt{\overline{l h}(1-\bar{l})(1-\bar{h})}} \\
& =\frac{\sum_{i=1}^{N}\left(l_{i} h_{i}\right)-N \overline{l h}-N \overline{l h}+N \overline{l h}}{N \sqrt{\overline{l h}(1-\bar{l})(1-\bar{h})}} \\
& =\frac{\sum_{i=1}^{N}\left(l_{i} h_{i}\right)-N \overline{l h}}{N \sqrt{\overline{l h}(1-\bar{l}(1-\bar{h})}} .
\end{aligned}
$$

Using $\sum_{i=1}^{N}\left(l_{i} h_{i}\right)=$ TP and Eq. (10), some algebra yields

$$
\rho=\frac{\mathrm{TP} \cdot \mathrm{TN}-\mathrm{FP} \cdot \mathrm{FN}}{\sqrt{(\mathrm{TP}+\mathrm{FN})(\mathrm{TP}+\mathrm{FP})(\mathrm{TN}+\mathrm{FP})(\mathrm{TN}+\mathrm{FN})}} .
$$

If any of $\mathrm{TP}+\mathrm{FN}, \mathrm{TP}+\mathrm{FP}, \mathrm{TN}+\mathrm{FP}$, or $\mathrm{TN}+\mathrm{FN}$ are 0 , then $\rho$ is defined as 0 . Note that, since $\rho$ is a correlation, it is not affected by the large number of non-outliers.

The Matthews Correlation is often used in Bioinformatics to assess the performance of classifiers, see, e.g. Shen and Chou (2007); Mondal et al. (2006); Kandaswamy et al. (2012).

\subsubsection{Relative Information score}

The Relative Information score was proposed by Kononenko and Bratko (1991) and relies on ideas from the Information Theory field. In this section we derive an expression for the Relative Information score based on the confusion matrix. Suppose we consider one particular point, then a priori we can compute the probability that it is an outlier from the confusion matrix

$$
\mathbb{P}(\text { outlier in high dimension })=\frac{\mathrm{TP}+\mathrm{FN}}{N} .
$$


After DR, we can compute this same probability for the same point as

$$
\mathbb{P}(\text { outlier in low dimension } \mid \text { outlier in high dimension })=\frac{\mathrm{TP}}{\mathrm{TP}+\mathrm{FN}} .
$$

Kononenko and Bratko (1991) argue that any well-performing classifier (DRmethods) should at least result in a confusion table with $\frac{\mathrm{TP}}{\mathrm{TP}+\mathrm{FN}}>\frac{\mathrm{TP}+\mathrm{FN}}{N}$, otherwise it has lost information from the original data. This forms the basis for their Relative Information score.

We introduce some notation and denote by $\mathbb{P}\left(C_{i}=c\right)$ the probability that point $i$ in the data set has class $c$, with $c=1$ indicating that it is an outlier in high dimension, and $c=0$ that it is a non-outlier. From the confusion matrix, we know that

$$
\begin{aligned}
& \mathbb{P}\left(C_{i}=1\right)=\frac{\mathrm{TP}+\mathrm{FN}}{N} \\
& \mathbb{P}\left(C_{i}=0\right)=\frac{\mathrm{FP}+\mathrm{TN}}{N} .
\end{aligned}
$$

After DR each point is again an outlier or non-outlier, but this time in low dimension. We denote the probability that point $i$ has class $c$, given that it also had class $c$ in high dimension, by $\mathbb{P}\left(C_{i}^{\prime}=c \mid C_{i}=c\right)$. From the confusion matrix, we find that

$$
\begin{aligned}
& \mathbb{P}\left(C_{i}^{\prime}=1 \mid C_{i}=1\right)=\frac{\mathrm{TP}}{\mathrm{TP}+\mathrm{FN}} \\
& \mathbb{P}\left(C_{i}^{\prime}=0 \mid C_{i}=0\right)=\frac{\mathrm{TN}}{\mathrm{FP}+\mathrm{TN}} .
\end{aligned}
$$

Kononenko and Bratko (1991) measure the amount of information (as defined by Shannon (1948)) necessary to correctly classify point $i$ as

$$
-\log _{2}\left(\mathbb{P}\left(C_{i}^{\prime}=c \mid C_{i}=c\right)\right) .
$$

They then give a positive score for a DR-methods that satisfies $\mathbb{P}\left(C_{i}^{\prime}=c \mid C_{i}=c\right)>\mathbb{P}\left(C_{i}=c\right)$ : 


$$
\log _{2}\left(\mathbb{P}\left(C_{i}^{\prime}=c \mid C_{i}=c\right)\right)-\log _{2}\left(\mathbb{P}\left(C_{i}=c\right)\right) .
$$

Some algebra shows that this is indeed a positive score. If $\mathbb{P}\left(C_{i}^{\prime}=c \mid C_{i}=c\right)<\mathbb{P}\left(C_{i}=c\right)$ the score is

$$
\log _{2}\left(1-\mathbb{P}\left(C_{i}=c\right)\right)-\log _{2}\left(1-\mathbb{P}\left(C_{i}^{\prime}=c \mid C_{i}=c\right)\right),
$$

which is negative. When $\mathbb{P}\left(C_{i}^{\prime}=c \mid C_{i}=c\right)=\mathbb{P}\left(C_{i}=c\right)$ the score is defined as 0 . The total score $I$ of a DR-methods is then

$$
\begin{aligned}
I & =\sum_{i=1}^{N} \mathbb{1}_{\left\{\mathbb{P}\left(C_{i}^{\prime}=c \mid C_{i}=c\right)>\mathbb{P}\left(C_{i}=c\right)\right\}} \cdot\left[\log _{2}\left(\mathbb{P}\left(C_{i}^{\prime}=c \mid C_{i}=c\right)\right)-\log _{2}\left(\mathbb{P}\left(C_{i}=c\right)\right)\right] \\
& +\mathbb{1}_{\left\{\mathbb{P}\left(C_{i}^{\prime}=c \mid C_{i}=c\right)<\mathbb{P}\left(C_{i}=c\right)\right\}} \cdot\left[\log _{2}\left(1-\mathbb{P}\left(C_{i}=c\right)\right)-\log _{2}\left(1-\mathbb{P}\left(C_{i}^{\prime}=c \mid C_{i}=c\right)\right)\right] .
\end{aligned}
$$

Usually, when comparing classifiers $I$ is reported relative to the expected information $E$ needed to correctly classify each point:

$$
E=-\sum_{i=1}^{N} \mathbb{P}\left(C_{i}^{\prime}=c \mid C_{i}=c\right) \cdot \log _{2}\left(\mathbb{P}\left(C_{i}^{\prime}=c \mid C_{i}=c\right)\right) .
$$

The Relative Information score $I_{r}$ is then

$$
I_{r}=\frac{I}{E} \cdot 100 \%
$$

Note that $I_{r}$ can become negative because $I$ can also be negative. Inserting Eqs. (14)-(18) into Eqs. (20) and (21) yields an expression in terms of the elements of the confusion matrix.

\section{Data sets}

In the previous sections we described setup of our experiments, the DR-techniques, and how we measure their performance. The experiments use three real-world data sets which we describe here, 


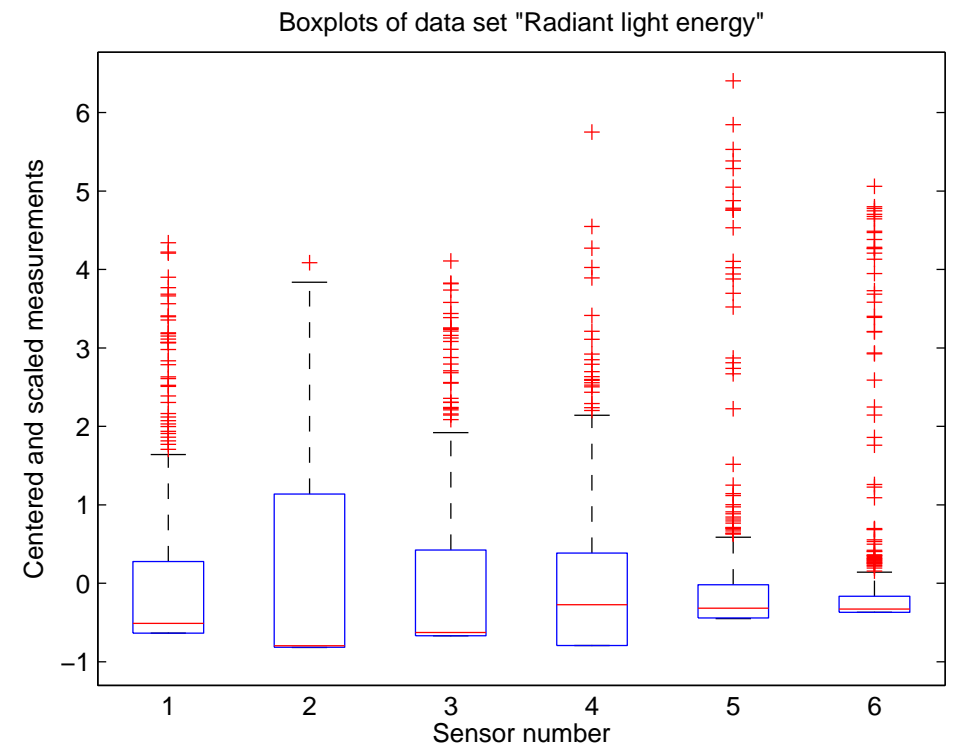

Figure 3. Boxplots of the six sensors in the "Radiant light energy" data set

\subsection{Radiant light energy measurements}

The measurements in this data set are from sensors deployed in several office buildings in New York City, as part of Columbia University's EnHANTs project. The sensors measure irradiance (radiant light energy), and this data set contains values measured during about one year.

Figure 3 shows boxplots of each of the six sensors in this data set, with the measurements centered and scaled as discussed in Section 3. Each boxplot reflects the distribution of the 500 measurements by one sensor, and highlights possible outliers. Each sensor contains 40-60 possible outliers, except for the second sensor which has just one. The Peeling algorithm from Section 3.1 will select which of these points we will use as outliers in our experiments. Note also that the median of each sensor's values (except sensor 4) is close to the .25 quantile, indicating that those distributions are skewed towards the smaller values.

More detailed information on the data set can be found in Gorlatova et al. (2011), or from the CRAWDAD website (Gorlatova et al. (2011)) where the data can be downloaded. For computational reasons, we do not use all the data for the experiments in this paper, but select 500 random measurements from each of the six sensors. 


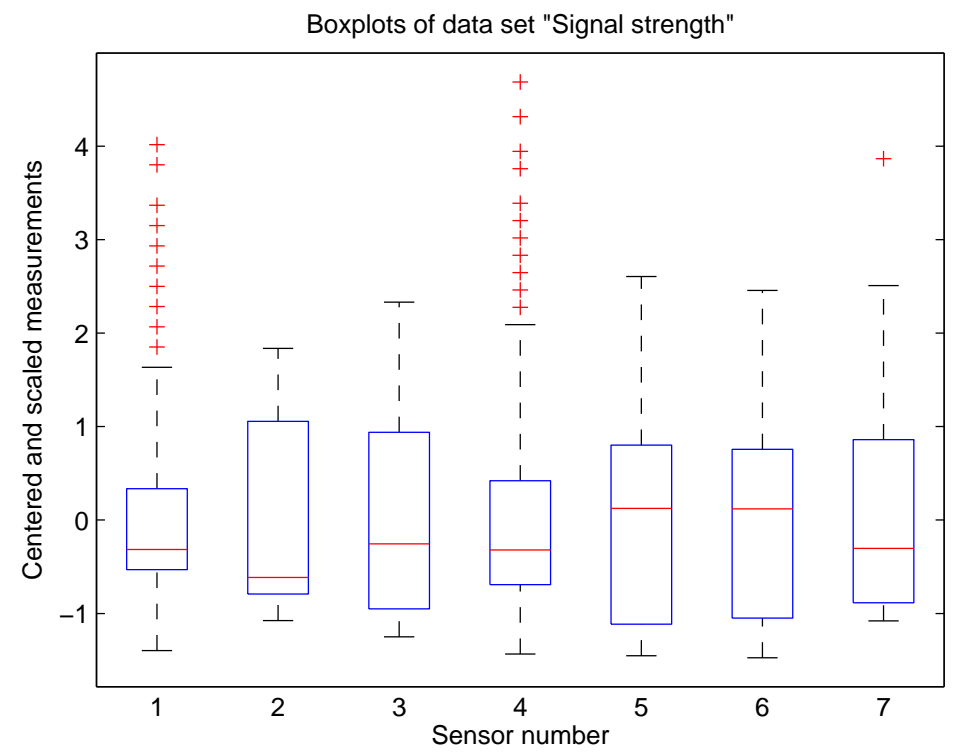

Figure 4. Boxplots of the seven sensors in the "Signal strength" data set

\subsection{Signal strength data}

This data originates from a WSN deployed in a library building, where sensors measure radio frequency energy level (RSSI) on all 802.15.4 channels in the 2.4 $\mathrm{GHz}$ ISM Band. In essence, RSSI is an indication of the power level of a signal received by the antenna on the sensor node. The building has several collocated Wi-Fi networks in normal operation that cause interference, so the WSN is used to monitor the signal strengths on one location in this Wi-Fi network. The WSN consists of 16 sensor nodes (each monitoring a single Wi-Fi channel) of which we used only 7, because the performance of convex hull algorithm in Onion Peeling decreases significantly for dimensions higher than 8 (see Barber et al. (1996)). Again, we took 500 randomly selected measurements of each node to form this data set.

The boxplots of the sensor values in this data set are shown in Figure 4. In contrast to "Radiant light energy" data set, the measurements of the sensors in the "Signal strength" data set contain fewer possible outliers and are more evenly distributed. All possible outliers are positive values, corresponding to a strong incoming Wi-Fi signal.

More details about the data can be found in Noda et al. (2011), or in the CRAWDAD repository (Noda et al. (2012)). 


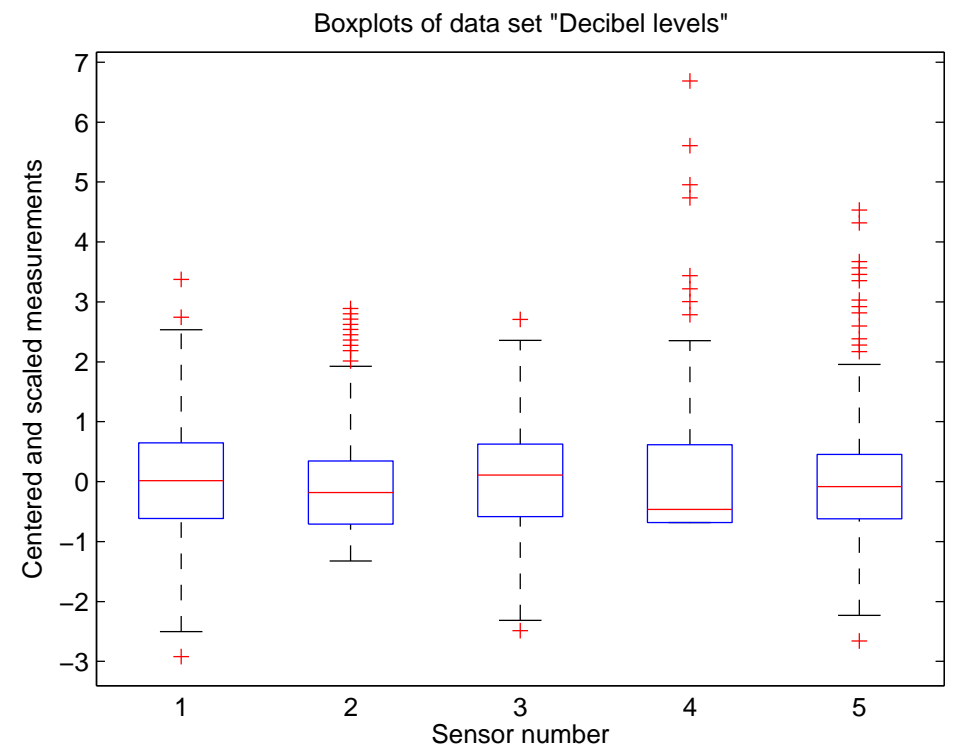

Figure 5. Boxplots of the five sensors in the "Decibel levels" data set

\begin{tabular}{l|ccc} 
DR-technique & Light & Signal & Decibel \\
\hline \hline PCA & 0.3333 & 0 & 0.3529 \\
MDS & 0.9091 & 0.8750 & 0.8889 \\
t-SNE & 0 & 0 & 0
\end{tabular}

Table 1. F1-score $(\in[0,1])$ of each combination of DR-technique and data set

\subsection{Decibel levels}

This data set consists of five sensors nodes deployed in a kindergarten, one in each room of a single-story building, that are used to monitor the indoor climate. Among other parameters, the nodes measure decibel levels, and report these regularly to a central base station. We took 500 measurements from each sensor on a day in May 2011 and included them in this data set.

Figure 5 shows that most sensors have fairly evenly distributed values, with several outliers on both sides of the median. However, kindergartens tend to be noisy rather than quiet, so most outliers are on the positive side of the median. 


\begin{tabular}{l|ccc} 
DR-technique & Light & Signal & Decibel \\
\hline \hline PCA & 0.3302 & -0.0149 & 0.3477 \\
MDS & 0.9119 & 0.8801 & 0.8926 \\
t-SNE & -0.0045 & -0.0061 & -0.0064
\end{tabular}

Table 2. Matthews Correlation $(\in[-1,1])$ for each combination of DR-technique and data set

\begin{tabular}{l|ccc} 
DR-technique & Light & Signal & Decibel \\
\hline \hline PCA & 0.7261 & -0.1295 & 0.6398 \\
MDS & 1.0509 & 0.9157 & 0.8971 \\
t-SNE & -0.0404 & -0.0244 & -0.0226
\end{tabular}

Table 3. Relative Information Score $(\in[-\infty, \infty])$ for each combination of DRtechnique and data set

\section{Results and discussion}

We apply the experimental setup of Section 3 to the DR-techniques of Section 2 and summarize the results in Tables 1-3. These tables contain the F1-score, Matthews Correlation, and Relative Information score for each combination of DR-technique and data set, where a high score implies that the technique preserves outliers well on that data set. The F1-scores in Table 1 show that MDS achieves the highest scores, with values more than twice as large as those of PCA on the first and third data set. The lowest possible score on all data sets is by t-SNE: an F1-score of 0. With the Matthews Correlation and Relative Information score in Tables 2 and 3 we see similar results: MDS consequently attains high scores, PCA performs reasonably well on the first and third data set, and t-SNE has overall low scores.

Since we reduce each data set to two dimensions, we can plot the resulting lowdimensional data set and inspect what happens with outliers after applying the three DR-methods. In Figures 6-8 we plot the low-dimensional version of the second data set "Signal strength" (in circles), with outliers in the original highdimensional data set marked with a triangle. Figure 6 shows that several of the outliers are mapped to the interior of the reduced data set by PCA. In contrast, the low-dimensional data set created by MDS shows all high-dimensional outliers close to the boundary. Lastly, t-SNE maps all outliers to the interior of the lowdimensional data set, which illustrates its low scores.

By analysing the objective of the three DR-techniques, we can explain the observed differences in performance. Firstly, PCA is a technique that focuses on preserving variance, so it will only preserve outliers if they happen to be in a direction of high variance. Figure 1 from the introduction provides another 
illustration of what can happen to an outlier that is in a direction with low variance. The figure corresponds to reducing a two-dimensional data set to one dimension (the line) with PCA, and clearly shows how the top-left outlier ends up in the center of the reduced data set.

MDS optimizes the squared stress optimization criterion in Eq. (4), which includes the term $\left\|\mathbf{x}_{i}-\mathbf{x}_{j}\right\|_{M}$. This term is the distance between two points $\mathbf{x}_{i}$ and $\mathbf{x}_{j}$, which is typically very large when one of the points is an outlier. The criterion uses these distances to the power 4 , so the outliers have a large effect on the squared stress criterion. Hence, minimizing these distances has a massive positive effect when on this criterion and thus MDS preserves outliers well.

t-SNE optimizes the Kullback-Leibler divergence (8), which attaches high costs to nearby points in high dimensional space (large $p_{i j}$ ) that are mapped to far away points in low dimensional space (small $q_{i j}$ ). Hence, nearby points in high dimensional space are kept nearby in low dimensional space. This does not hold for points that are far away in high dimensional space - outliers, which have low $p_{i j}$ - as they are mapped to nearby points (with high $q_{i j}$ ) with very low costs. So t-SNE tries to keep nearby points nearby and is therefor more suitable for preserving clusters than for preserving outliers.

Also, t-SNE has some computational complexities. The Kullback-Leibler divergence in Eq. (8) is a non-linear function of the low-dimensional points $\mathbf{y}_{i}$ and can have several local minimums. Minimizing Eq. (8) is done by choosing a random starting point for the $\mathbf{y}_{i}$, followed by a number of optimization steps using, e.g., a Gradient Descent approach. The optimization stops when it reaches a (possibly local) minimum, and returns its latest values for the $\mathbf{y}_{i}$. Hence, the low-dimensional points depend on the starting point chosen for the optimization algorithm, and thus its performance with respect to outlier preservation also depend on the starting values of the $\mathbf{y}_{i}$. The last rows of Tables 1-3 show the score for one particular starting point, but we repeated the experiments with t-SNE for various starting points. We discovered no correlation between the minimized value of the Kullback-Leibler divergence and the achieved performance score, so the results reported in Tables 1-3 are representative for the performance of t-SNE.

From the analysis above we see that from the three selected methods, MDS achieves the highest scores and is best capable of preserving outliers. However, it is not necessarily the best DR-technique available, since many others exist in literature. In particular, the class of supervised DR-techniques (PCA, MDS, t-SNE are unsupervised) might provide methods with better performance than MDS. These techniques aim to reduce dimensionality while simultaneously trying to retain "sufficient information" for a classification task (which, in our case, would be retaining outliers). Hence, they could be applied to the scenario in this paper, and possibly have good performance. Nevertheless, supervised DRtechniques are not included here, because we assume that the DR-techniques 


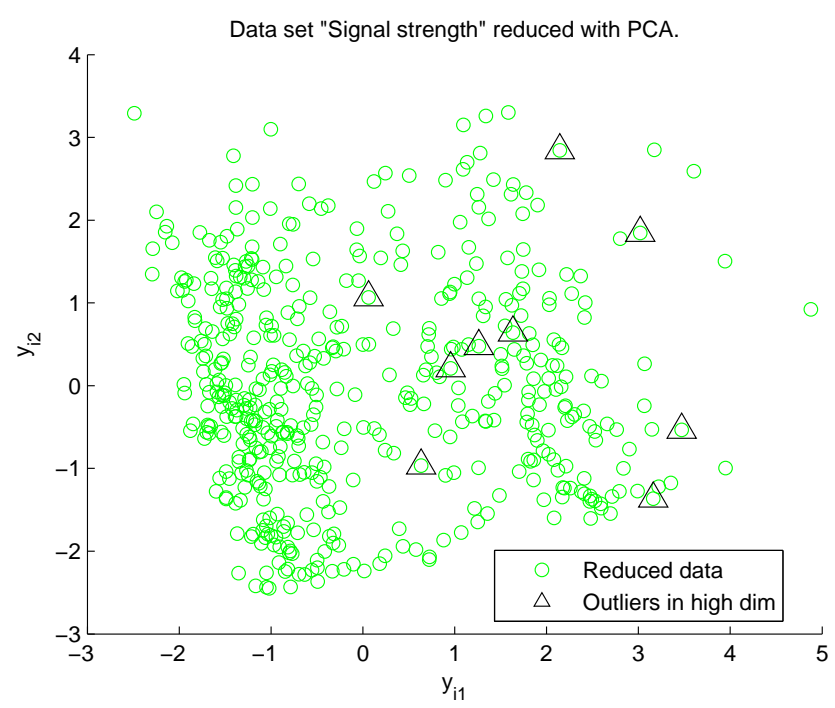

Figure 6. Data set "Decibel levels" after dimensionality reduction with PCA (circles). The triangles mark the outliers that were found in the original high-dimensional data set

have no apriori knowledge about the outliers, and thus they are not suitable for this paper. Readers interested in supervised DR-techniques are referred to, e.g., Shyr et al. (2010).

The performance measures in this paper are all based on the elements of the confusion matrix, which do not contain information about whether a point is a 'large' or 'small' outlier. Hence, with these scores we will not be able to, e.g., find out which outlier has the large affect on a score. This 'binary' view of an outlier is, however, important for the scenario in the current paper. Our motivation comes from applications where it is of critical importance to correctly identify an outlier after DR. If an outlier is no longer an outlier after DR, then it is useless for the application. Nevertheless, if this 'binary' approach can be relaxed from the point of view of the application, other scores might be more appropriate (see, e.g., Bradley (1997)).

\section{Conclusions and recommendations}

In this paper we described three well-known Dimensionality Reduction techniques (Principal Component Analysis, Multidimensional Scaling, and t-Stochastic Neighbourhood Embedding) and analysed how well they are capable of preserving outliers. Based on three different scores (F1-score, Matthews Correlation, 


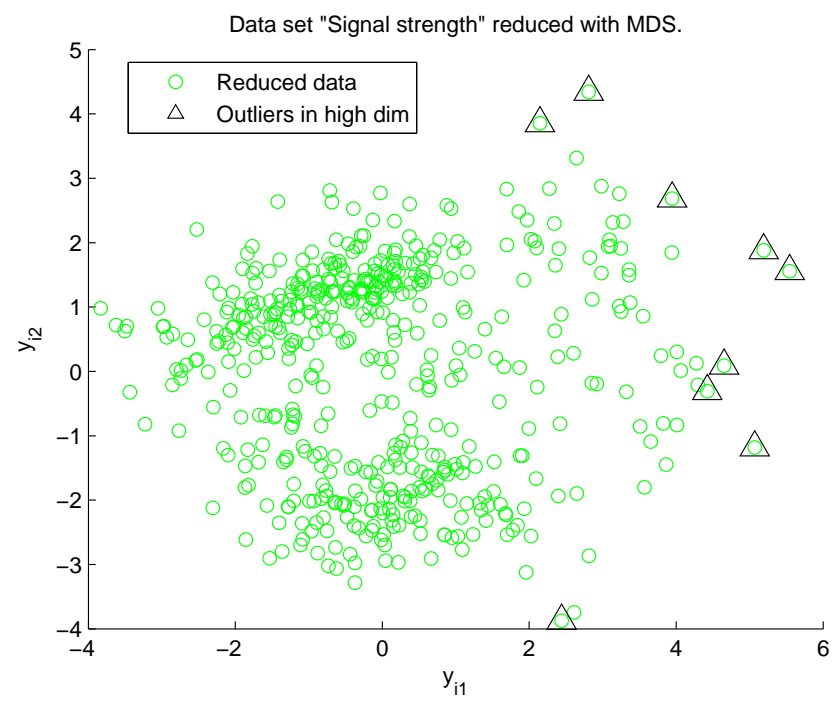

Figure 7. Data set "Decibel levels" after dimensionality reduction with MDS (circles). The triangles mark the outliers that were found in the original high-dimensional data set

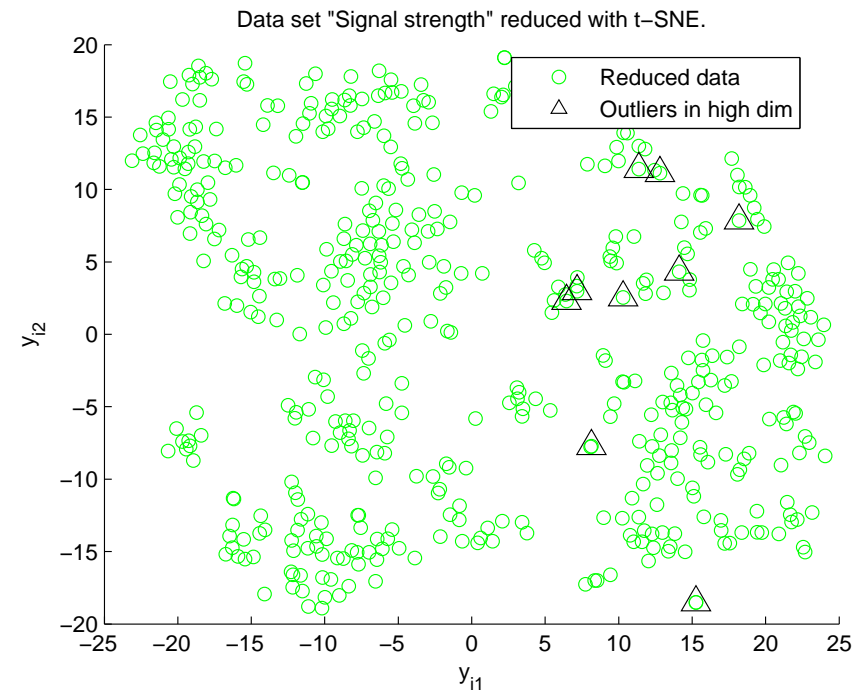

Figure 8. Data set "Decibel levels" after dimensionality reduction with t-SNE (circles). The triangles mark the outliers that were found in the original high-dimensional data set 
and Relative Information score), and using three real-world data sets, we assessed the performance of each method on each data set.

The resulting analysis shows that, among the three described DR-methods, Multidimensional Scaling is best at preserving outliers. It consequently achieves the highest scores, and performs significantly better than both Principal Component Analysis and t-Stochastic Neighbourhood Embedding. In the discussion, we explain that this difference in performance is caused by the specific objectives of the techniques: PCA tries to preserve variance, MDS preserves large distances (i.e. outliers), and t-SNE preserves clusters. In general, we recommend that the dimensionality reduction technique is chosen with the intended application in mind. For outlier detection MDS is a good choice, for preserving variance PCA is the best choice, and for preserving clusters t-SNE is a good choice.

Future research includes investigating specific types of Dimensionality Reduction (e.g. supervised DR-methods, real-time DR-methods), and how they are affected by outliers.

\section{Acknowledgements}

This work is based on a report by Onderwater (2010) of an internship at the Fraud Detection Expertise Center at VU University Amsterdam. The current paper is part of the project RRR (Realisation of Reliable and Secure Residential Sensor Platforms) of the Dutch program IOP Generieke Communicatie, number IGC1020, supported by the Subsidieregeling Sterktes in Innovatie. We would like to thank Arnoud den Boer (CWI, VU University) for his feedback on the drafts of this document, and Munisense B.V. (Leiden, The Netherlands) for providing one of the data sets.

\section{References}

Agyemang, M., K. Barker, and R. Alhajj (2006). A comprehensive survey of numeric and symbolic outlier mining techniques. Intelligent Data Analysis 10(6), $521-538$.

Barber, C. B., D. P. Dobkin, and H. Huhdanpaa (1996). The quickhull algorithm for convex hulls. ACM Transactions on Mathematical Software 22(4), $469-483$.

Becker, R. A., C. Volinsky, and A. R. Wilks (2010). Fraud detection in telecommunications: History and lessons learned. Technometrics $\underline{52}(1), 20-33$. 
Bradley, A. P. (1997). The use of the area under the ROC curve in the evaluation of machine learning algorithms. Pattern recognition 30(7), 1145-1159.

Cao, H., D. H. Hu, D. Shen, D. Jiang, J.-T. Sun, E. Chen, and Q. Yang (2009). Context-aware query classification. In Proceedings of the 32nd international ACM SIGIR conference on Research and development in information retrieval, pp. 3-10.

Carreira-Perpinán, M. A. (1997). A review of dimension reduction techniques. Department of Computer Science. University of Sheffield. Tech. Rep. CS-96-09, 1-69.

Chakrabarti, K. and S. Mehrotra (2000). Local dimensionality reduction: A new approach to indexing high dimensional spaces. In Proceedings ofthe 26th VLDB Conference, pp. 89-100.

Dai, J. J., L. Lieu, and D. Rocke (2006). Dimension reduction for classification with gene expression microarray data. Statistical applications in genetics and $\underline{\text { molecular biology }} \underline{5}(1)$.

Escalante, H. J. (2005). A comparison of outlier detection algorithms for machine learning. In CIC-2005 Congreso Internacional en Computacion.

Fodor, I. (2002). A survey of dimension reduction techniques. Technical Report UCRL-ID-148494, Lawrence Livermore National Laboratory.

Garg, V. K. and M. N. Murty (2009). Feature subspace SVMs (FS-SVMs) for high dimensional handwritten digit recognition. International Journal of Data Mining, Modelling and Management 1(4), 411.

Ghodsi, A. (2006). Lecture notes of lecture 9 of the course "Data visualization" (STAT 442). Technical report, University of Waterloo.

Gogoi, P., D. Bhattacharyya, B. Borah, and J. K. Kalita (2011). A survey of outlier detection methods in network anomaly identification. Comput. J. 54(4), 570-588.

Gorlatova, M., A. Wallwater, and G. Zussman (2011). Networking low-power energy harvesting devices: Measurements and algorithms. In INFOCOM, 2011 Proceedings IEEE, pp. $1602-1610$.

Gorlatova, M., M. Zapas, E. Xu, M. Bahlke, I. Kymissis, and G. Zussman (2011). CRAWDAD data set columbia/enhants (v. 2011-04-07). Published: Downloaded from http://crawdad.cs.dartmouth.edu/columbia/enhants.

Gupta, R. and R. Kapoor (2012). Comparison of graph-based methods for non-linear dimensionality reduction. International Journal of Signal and Imaging Systems Engineering 5(2), 101-109. 
Hahn, L. W., M. D. Ritchie, and J. H. Moore (2003). Multifactor dimensionality reduction software for detecting gene-gene and gene-environment interactions. Bioinformatics 19(3), 376-382.

Härdle, W. K. and L. Simar (2012). Applied Multivariate Statistical Analysis. Springer.

Harmeling, S., G. Dornhege, D. Tax, F. Meinecke, and K.-R. Müller (2006). From outliers to prototypes: Ordering data. Neurocomputing 69(13-15), $1608-1618$.

Hinton, G. and S. Roweis (2002). Stochastic neighbor embedding. In Advances in Neural Information Processing Systems 15, pp. 833-840. MIT Press.

Hodge, V. and J. Austin (2004). A survey of outlier detection methodologies. Artif. Intell. Rev. 22(2), 85-126.

Johnson, R. and D. Wichern (2002). Applied Multivariate Statistical Analysis. Prentice Hall.

Kandaswamy, K. K., G. Pugalenthi, K.-U. Kalies, E. Hartmann, and T. Martinetz (2012). EcmPred: prediction of extracellular matrix proteins based on random forest with maximum relevance minimum redundancy feature selection. Journal of theoretical biology.

Kline, D. M. and C. S. Galbraith (2009). Performance analysis of the bayesian data reduction algorithm. International Journal of Data Mining, Modelling and Management 1(3), 223.

Kohonen, T. (2001). Self-organizing maps (3rd ed.), Volume 30. Springer.

Kononenko, I. and I. Bratko (1991). Information-based evaluation criterion for classifier's performance. Machine Learning $\underline{6}(1), 67-80$.

Lattin, J., D. Carroll, and P. Green (2003). Analyzing Multivariate Data. Thomson Learning.

Li, K.-C. (1992). On principal hessian directions for data visualization and dimension reduction: Another application of stein's lemma. Journal of the American Statistical Association 87(420), 1025-1039.

Maalouf, M. and T. B. Trafalis (2011). Rare events and imbalanced datasets: an overview. International Journal of Data Mining, Modelling and Management $3(4), 375$.

Mahalanobis, P. C. (1936). On the generalized distance in statistics. In Proceedings of the national institute of sciences of India, Volume 2, pp. 49-55.

Martins, B., I. Anastácio, and P. Calado (2010). A machine learning approach for resolving place references in text. In M. Painho, M. Y. Santos, and H. Pundt (Eds.), Geospatial Thinking, Number 0 in Lecture Notes in Geoinformation and Cartography, pp. 221-236. Springer Berlin Heidelberg. 
Matthews, B. (1975). Comparison of the predicted and observed secondary structure of t4 phage lysozyme. Biochimica et Biophysica Acta (BBA) Protein Structure 405(2), 442-451.

Mondal, S., R. Bhavna, R. Mohan Babu, and S. Ramakumar (2006). Pseudo amino acid composition and multi-class support vector machines approach for conotoxin superfamily classification. Journal of Theoretical Biology 243(2), 252-260.

Muñoz, A. and J. Muruzábal (1998). Self-organizing maps for outlier detection. Neurocomputing $18(1-3), 33-60$.

Nguyen, H. V., V. Gopalkrishnan, H. Liu, H. Motoda, R. Setiono, and Z. Zhao (2010). Feature extraction for outlier detection in highdimensional spaces. In The 4th Workshop on Feature Selection in Data Mining.

Noda, C., S. Prabh, M. Alves, C. A. Boano, and T. Voigt (2011). Quantifying the channel quality for interference-aware wireless sensor networks. SIGBED Rev. ${ }^{8}(4), 43-48$.

Noda, C., S. Prabh, M. Alves, T. Voigt, and C. A. Boano (2012). CRAWDAD data set cister/rssi (v. 2012-05-17). Published: Downloaded from http://crawdad.cs.dartmouth.edu/cister/rssi.

Onderwater, M. (2010). Detecting unusual user profiles with outlier detection techniques. M.Sc. thesis, http://tinyurl.com/vu-thesis-onderwater.

Pearson, K. (1901). On lines and planes of closest fit to systems of points in space. Philosophical Magazine Series 6 2(11), 559-572.

Phua, C., V. Lee, K. Smith, and R. Gayle (2005). A comprehensive survey of data mining-based fraud detection research. Technical report, Monash University.

Powers, D. M. W. (2011). Evaluation: From precision, recall and f-measure to ROC., informedness, markedness \& correlation. Journal of Machine Learning Technologies 2 (1), 37-63.

Ravi, V. and C. Pramodh (2010). Non-linear principal component analysisbased hybrid classifiers: an application to bankruptcy prediction in banks. International Journal of Information and Decision Sciences 2(1), 50 .

Sha, F. and F. Pereira (2003). Shallow parsing with conditional random fields. In Proceedings of the 2003 Conference of the North American Chapter of the Association for Computational Linguistics on Human Language Technology-Volume 1, pp. 134-141.

Shannon, C. E. (1948). A mathematical theory of communication. Bell system technical journal 27. 
Shen, H.-B. and K.-C. Chou (2007). Using ensemble classifier to identify membrane protein types. Amino Acids 32(4), 483-488.

Shyr, A., R. Urtasun, and M. I. Jordan (2010). Sufficient dimension reduction for visual sequence classification. In 2010 IEEE Conference on Computer Vision and Pattern Recognition (CVPR), pp. 3610-3617.

Tabachnick, B. G. and L. S. Fidell (2001). Using multivariate statistics. Allyn and Bacon.

Tenenbaum, J. B., V. d. Silva, and J. C. Langford (2000). A global geometric framework for nonlinear dimensionality reduction. Science 290(5500), 23192323.

Tsai, F. S. (2012). Dimensionality reduction framework for blog mining and visualisation. International Journal of Data Mining, Modelling and Management $\underline{4}(3), 267-285$.

UmaMaheswari, P. and M. Rajaram (2009). Principal component analysisbased frequent pattern evaluation on the object-relational data model of a cricket match database. International Journal of Data Analysis Techniques and Strategies 1 (4), 364 .

Valstar, M. F., B. Jiang, M. Mehu, M. Pantic, and K. Scherer (2011). The first facial expression recognition and analysis challenge. In Automatic Face \& Gesture Recognition and Workshops (FG 2011), 2011 IEEE International Conference on, pp. 921-926.

Van der Maaten, L. (2009). Dimensionality reduction toolbox.

Van der Maaten, L. and G. Hinton (2008). Visualizing data using t-SNE. Journal of Machine Learning Research 9, 2579-2605.

Van der Maaten, L., E. Postma, and J. Van den Herik (2009). Dimensionality reduction: A comparative review. Technical Report 2009-005, Tilburg centre for Creative Computing, Tilburg university.

Yoon, S. (2003). Singular Value Decomposition \& Application.

Zhang, Y., N. Meratnia, and P. Havinga (2010). Outlier detection techniques for wireless sensor networks: A survey. IEEE Communications Surveys \& Tutorials 12 (2), 159-170.

Zhang, Y., N. Meratnia, and P. J. M. Havinga (2007). A taxonomy framework for unsupervised outlier detection techniques for multi-type data sets. Number TR-CTIT-07-79. Enschede: Centre for Telematics and Information Technology, University of Twente. 